Technological University Dublin

DƯBLIN

ARROW@TU Dublin

\title{
Quantification and Identification of Microproteinuria using Ultrafiltration and ATR-FTIR Spectroscopy
}

\author{
David Perezguaita \\ Technological University Dublin, david.perezguaita@tudublin.ie \\ Zack Richardson \\ Monash University \\ Phil Heraud \\ Monash University
}

See next page for additional authors

Follow this and additional works at: https://arrow.tudublin.ie/scschphyart

Part of the Medicine and Health Sciences Commons

\section{Recommended Citation \\ Perez-Guaita, D. et al. (2020) Quantification and Identification of Microproteinuria using Ultrafiltration and ATR-FTIR Spectroscopy, Anal. Chem, 2020 Feb 4;92(3):2409-2416. doi: 10.1021/acs.analchem.9b03081}

This Article is brought to you for free and open access by the School of Physics \& Clinical \& Optometric Science at ARROW@TU Dublin. It has been accepted for inclusion in Articles by an authorized administrator of ARROW@TU Dublin. For more information, please contact arrow.admin@tudublin.ie, aisling.coyne@tudublin.ie, gerard.connolly@tudublin.ie.

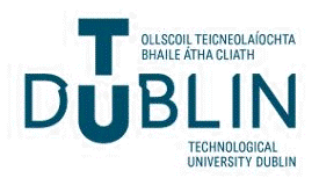


Authors

David Perezguaita, Zack Richardson, Phil Heraud, and Bayden Wood

This article is available at ARROW@TU Dublin: https://arrow.tudublin.ie/scschphyart/125 


\title{
Quantification and Identification of Microproteinuria using Ultrafiltration and ATR-FTIR Spectroscopy
}

David Perez-Guaita $^{* 1,2}$, Zack Richardson ${ }^{1}$, Philip Heraud ${ }^{1,3}$, Bayden Wood $^{* 1}$

${ }^{1}$ Centre for Biospectroscopy and School of Chemistry, Monash University, Clayton Campus, 3800, Victoria, Australia

${ }^{2}$ FOCAS Research Institute, Dublin Institute of Technology, D04, Dublin, Ireland

${ }^{3}$ Infection and Immunity Program, Monash Biomedicine Discovery Institute and Department of Microbiology, Monash University, Clayton Campus, 3800, Victoria, Australia

*Corresponding Authors: David.PerezGuaita@dit.ie,Bayden.Wood@monash.edu

\begin{abstract}
The presence of low amounts of specific proteins in urine can be an indicator of diagnosis and prognosis of several diseases including renal failure and cancer. Hence, there is an urgent need for Point-of-Care (PoC) methods, which can quantify microproteinuria levels (30-300 ppm) and identify the major proteins associated with the microproteinuria. In this study, we coupled ultracentrifugation with Attenuated Total ReflectanceFourier Transform Infrared (ATR-FTIR) to identify and quantify proteins in urine at low ppm levels. The process involves the pre-concentration of proteins from $500 \mu \mathrm{L}$ of urine using an ultrafiltration device. After several washings, the isolated proteins are dried onto the ATR crystal forming a thin film. Imaging studies showed that the absorbance of the protein bands was linear with the amount of mass deposited on the crystal. The methodology was first evaluated with artificial urine spiked with 30-300 ppm of albumin. The calibration showed acceptable linearity $\left(\mathrm{R}^{2}=0.97\right)$ and a limit of detection of $6.7 \mathrm{ppm}$. Linear relationships were also observed from urine of healthy subjects spiked with microproteinuria concentrations of albumin, immunoglobulin and haemoglobin giving a prediction error of the spiked concentration of $23 \mathrm{ppm}$. When multiple proteins were spiked into the real urine, multivariate analysis was able to decompose the dataset into the different proteins, but the multicomponent evaluation was challenging for proteins at low levels. Although the introduction of a pre-processing step reduces the PoC capability of the method, it largely increases its performance, showing great potential as a tool for the diagnosis and prognosis of several illnesses affecting urine proteic composition.
\end{abstract}




\section{Introduction}

Proteins are the major non-aqueous component of blood plasma, but in healthy patients their concentration in urine is minimal because they are filtered by the kidney. The presence of proteins in urine is associated with a wide range of conditions that can be differentiated by three ranges of concentrations, which are defined based on the volume of urine produced in 24 hours. Protein amounts within the normal range $\left(<30 \mathrm{mg} \mathrm{L}^{-1} \mathrm{or}\right.$ $<30 \mathrm{mg}$ of proteins $/ 24$ hours) are present in urine naturally. Values above $300 \mathrm{mg} \mathrm{L}^{-1}$ or $300 \mathrm{mg}$ of proteins $/ 24 \mathrm{~h}$ indicate macroproteinuria, a condition symptomatic of several diseases, such as renal and cardiac conditions. ${ }^{1}$ Protein values between 30 and $300 \mathrm{mg} \mathrm{L}^{-1}$ or between 30 and $300 \mathrm{mg} / 24$ hours are defined as microalbuminuria, which is also related to numerous conditions, including renal malfunction ${ }^{2}$ and cancer $^{3}$.

When microproteinuria is caused by albumin it is often referred to as "microalbuminuria". This parameter is especially important as a marker of diabetes mellitus and hypertension and is used for both, prognosis and diagnosis. ${ }^{4-6}$ Proteic analysis of urine is an emerging tool for the study and detection of several diseases, ${ }^{7,8}$ however, the tests are not routine and not suitable for PoC diagnosis. New methods should be quick, costeffective and simple to use in order to quantify proteins within the microproteinuria concentration range in urine (30-300 ppm based on the volume of urine produced in 24 hours). Ideally, methods should also identify the major proteins contained in urine to provide more diagnostic information about the cause of microproteinuria. In the clinical setting, regular urine dipsticks can detect protein concentrations only above the microporteinuria level. Microalbuminuria is evaluated using specific dipsticks such as the "Micral-Test". These strips are based on immunological antibodies highly specific for human albumin and are very suitable for PoC testing. On the other hand, they offer semi-quantitative results and only have a limited concentration range (20-200) resulting in low specificity ${ }^{9}$.

Fourier Transform Infrared (FTIR) spectroscopy has shown great potential in quantifying clinical parameters $^{10}$ and diagnosing disease ${ }^{11-13}$. Biochemical components absorb infrared light at specific wavelengths and the resulting spectrum can be related to the presence and concentration of biological molecules. FTIR spectroscopy is a versatile technique because the sample spectrum can be measured in a wide range of instruments, from high performance microscopes to portable spectrometers. The latter are becoming 
very popular because their suitability as PoC diagnostic tools. They can provide information about the major composition of the sample with minimum or no sample treatment. Attenuated Total Reflectance (ATR) is the preferred technique for PoC testing because it only requires depositing few microliters of the sample onto the ATR crystal. In urine, ATR-FTIR spectroscopy has been proposed for quantifying creatinine, urea, phosphate, uric acid, $\mathrm{pH}$ and sulphate ${ }^{14,15}$ and cystinuria ${ }^{16}$. The determination of albumin from untreated urine has also been investigated using FTIR spectroscopy, proven to be successful at high macroalbuminuria levels but clearly ineffective at microalbuminuria levels ${ }^{17}$, showing a "significant spread especially at low concentration values" $" 14$.

The main challenge in quantifying clinical parameters in urine using ATR is the lack of sensitivity. For example, in serum, studies indicate that the limited pathlength of ATR makes it difficult to quantify parameters below $20 \mathrm{mg} / \mathrm{dL}$ such as uric acid or creatinine ${ }^{10,18,19}$. In urine, low concentrated analytes such as exogenous compounds have been determined only after sample pre-treatment steps, which isolated the analyte from the rest of urine components and enabled the pre-concentration of the target compound on the surface of the crystal. For example solid-phase micro-extraction (SPME) and liquid-liquid micro extraction were used to detect ibuprofen ${ }^{20}$ and lidocaine ${ }^{21}$ at the low ppm range, respectively.

Recently, ultrafiltration devices have been used to separate proteins from metabolites in serum prior to infrared analysis. ${ }^{22}$ Reports indicate that measuring the low and high molecular fraction improved the determination of glucose $\mathrm{e}^{23}$ and $\mathrm{IgG}^{24}$, respectively. The aim of this study was to develop a method for the quantification and identification of microproteinuria by coupling ultrafiltration with ATR-FTIR for the quantitative measurement of dried urine films. Artificial urine was used to establish the Limit of Detection (LOD) and limit of quantification (LOQ) of the technique, because in all the volunteer urine analyzed there was some evidence of protein. This is well established in the literature, where studies on healthy volunteers always show low levels of protein $(<30 \mathrm{mg} \mathrm{L}-1 \mathrm{or}<30 \mathrm{mg}$ of proteins $/ 24$ hours $) .{ }^{1}$ The methodology was then evaluated using urine from volunteers spiked with albumin, immunoglobulin and hemoglobin at microproteinuria levels. Dry films were analysed using imaging techniques to evaluate the morphology and investigate what areas of the dried film were best suited for quantification.

\section{Methods}


Urine was collected from healthy volunteers $(\mathrm{N}=6$, ethical approval by Monash University Committee: 2019-21431-34708). Prior to treatment, it was kept at room conditions until the temperature of the sample stabilized. All samples and artificial urine were filtered using $0.22 \mu \mathrm{m}$ filters from Corning incorporated (Corning, USA) in order to remove cells or other impurities. Artificial urine was synthesised following the same protocol as in Worramongkona et $\mathrm{al}^{25}$. Standards of $\mathrm{CaCl}_{2}$, Creatinine, $\mathrm{KCl}, \mathrm{MgSO}_{4}$, Sodium Citrate, $\mathrm{NaCl}, \mathrm{NaHCO}_{3}, \mathrm{Na}_{2} \mathrm{HPO}_{4}, \mathrm{NaH}_{2} \mathrm{PO}_{4}, \mathrm{Na}_{2} \mathrm{SO}_{4}, \mathrm{NH}_{4} \mathrm{Cl}$, Urea and Uric acid used for creating artificial urine were obtained from Merck (St. Louis, USA). The $\mathrm{pH}$ of the artificial urine was adjusted to $6.0 \mathrm{using} \mathrm{HCl}$. Albumin and hemoglobin standards were purchased from Merck (St. Louis, USA) and immunoglobulin standards were purchased from Lee Biosolutions (Maryland, USA)

\section{Protein pre-concentration and extraction}

$500 \mu \mathrm{L}$ of spiked urine or artificial urine was transferred to a $0.5 \mathrm{~mL}$ Amicon $\subset$ ultra-filtration device with a nominal molecular weight limit of $10 \mathrm{kDa}$ purchased from Merck (St. Louis, USA). Samples were centrifuged at $14000 \mathrm{~g}$ for 15 minutes. Clean-up of the extract was carried out in the same device. $450 \mu \mathrm{L}$ of ultrapure water was added to the pre-concentrated urine extract and the mixed solution was centrifuged at $14000 \mathrm{~g}$ for 15 minutes. The washing process was repeated 3 times. Prior to the measurements, the preconcentration devices were washed with ultrapure water following the instructions of the manufacturer in order to minimize glycerol contamination.

\section{Dry film measurement and data treatment}

$0.8 \mu \mathrm{L}$ of the extracts were deposited on the ATR crystal using a standard $0.1-1 \mu \mathrm{L}$ pipette. The deposited volume was dried under a gentle air-flow until no signal of water was found in the spectra (1-2 minutes), which was monitored using the $\mathrm{O}-\mathrm{H}$ stretching $\left(3200 \mathrm{~cm}^{-1}\right)$ and bending bands $\left(1600 \mathrm{~cm}^{-1}\right)$ from liquid water. An FTIR ALPHAC spectrometer from Bruker (Ettlingen, Germany) was used to acquire the spectra. The instrument was equipped with a single bounce diamond ATR crystal. Spectra were measured at $4 \mathrm{~cm}^{-1}$ spectral resolution, co-averaging 40 interferograms (40 seconds). A background spectrum of the clean empty cell was acquired every hour, or immediately after observing water vapour bands, by co-adding 100 interferograms. 
Each spectrum was computed by averaging 3 depositions (120 scans in total). The spectrometer was controlled using OPUS software from Bruker (Ettlingen, Germany). Data analysis, including Principal Component Analysis (PCA) and integration of the spectral bands were carried out using in-house written functions in Matlab from Mathworks (Natick, USA) and the PLS toolbox from Eigenvector (Manson, USA). Before integration of amide I and II bands spectra were smoothed using a Savitzky-Golay filter with a 21-points window width over the spectral range $1507-1700 \mathrm{~cm}^{-1}$, using baselines fitted on the $1492-1503 \mathrm{~cm}^{-1}$ and 1720 $1750 \mathrm{~cm}^{-1}$ regions. Multivariate Curve Resolution- Alternate Least Squares (MCR-ALS) was carried out using the interface developed by Roma Tauler's group. ${ }^{26}$

Imaging analysis of the Dry films.

Dry films were deposited on an ATR crystal which was detached from an instrument and placed on the stage of an Alpha 300 Raman confocal microscope from Witec (Ulm, Germany). This microscope was used for acquiring visible images using a 10x objective. Topography images were measured by employing an Atomic Force Microscope (AFM) nano-IR II from Anasys (Santa Barbara, USA).

\section{Results}

\section{Evaluation of the pre-concentration and purification processes}

Initially we studied the composition of urine from a healthy volunteer and inevstiagted pre-concentrated extracts by acquiring ATR-FTIR spectra from the dried films. Figure 1a (blue spectrum) shows the spectrum of raw urine before sample treatment and after the pre-concentration and washing steps. The spectrum of untreated urine is dominated by the signals of urea and creatinine, which are the major components of urine. These prominent bands are found at $1470 \mathrm{~cm}^{-1}$ and $1618 \mathrm{~cm}^{-1}$ and are assigned to the $v_{\text {as }}(\mathrm{CN})$ and $\delta(\mathrm{NH})^{27}$
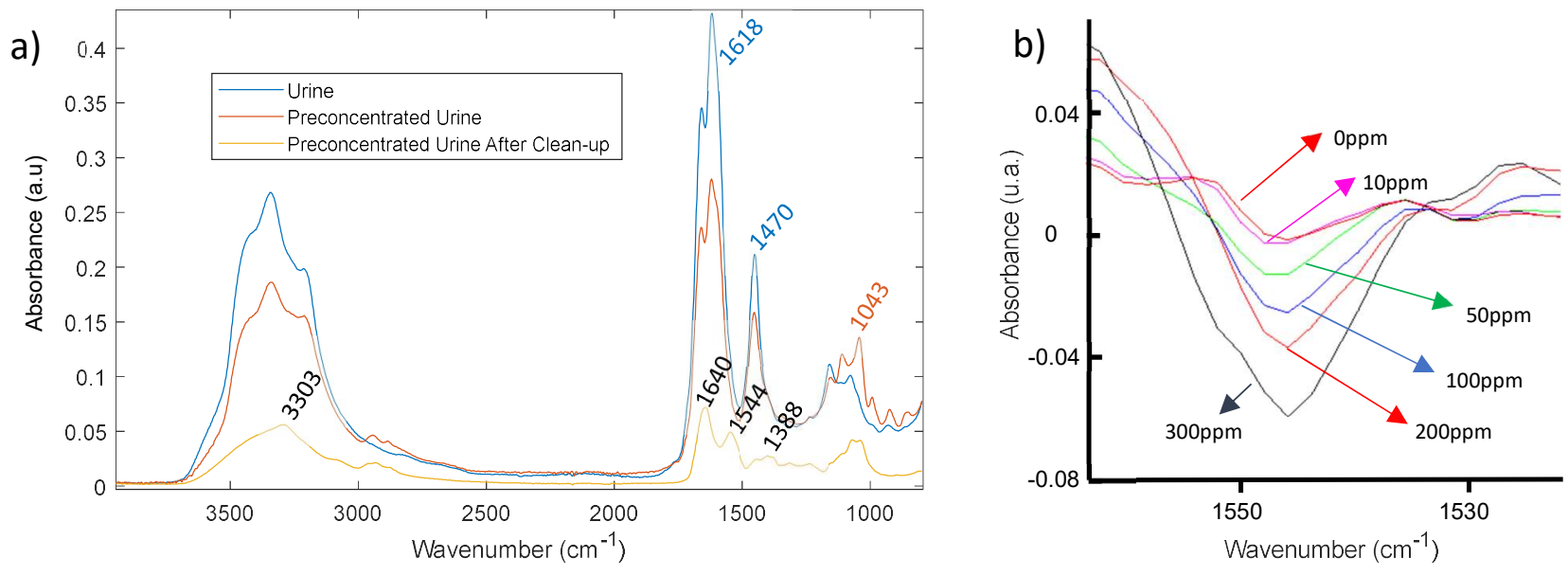
modes of urea, respectively. These peaks also overlap with $v_{\mathrm{as}}(\mathrm{CN})$ and $\delta(\mathrm{NH})$ modes from creatinine. A visual inspection of the amides I and II region does not reveal any signal of proteins. In healthy urine, concentrations of urea and creatinine are found in the 4000-12000 ppm and 500-2000 ppm ranges, respectively (See Scheme S1) for a graphical comparison of metabolites and protein levels in urine). These major metabolites are three orders of magnitude more concentrated than proteins at microproteinuria levels (30-300 ppm), which implies that proteins will be diluted in the urea and creatinine film and will be hardly detectable at microproteinuria or lower levels, as in the case of the healthy volunteer. This is in good agreement with results from Shaw et $\mathrm{al}^{17}$, where the predicted vs reference figure shows that multivariate analysis was able to quantify proteins only above 10000 ppm

Figure 1. (a) Typical spectra of dried films from a urine sample before treatment and after pre-concentration and purification steps. Sample was collected from a healthy volunteer. (b) Second derivative spectra of preconcentrated urine spiked with different levels of albumin (ppm).

After the pre-concentration step, protein levels are multiplied by the pre-concentration factor (considering initial and final volumes, the pre-concentration factor was roughly 22), whereas the level of other metabolites remains the same. Microproteinuria pre-concentrated levels would correspond roughly to $600-6000 \mathrm{ppm}$ $(<600 \mathrm{ppm}$ for the healthy volunteers). At first sight, the spectrum of the pre-concentrated urine remains similar to the spectrum of the raw urine and no signal of protein was found (refer to orange spectra in Figure 1a). Bands in the range $1100-900 \mathrm{~cm}^{-1}$, which appear in the pre-concentrated urine, are assigned to alcohol from the glycerol and are used as preservatives in the membrane. However, in another experiment, urine was spiked with low levels of albumin (10-300 ppm) to investigate microproteinuria concentrations. The spectra of the dried film of the pre-concentrated samples are shown in Figure 1b. The second derivative revealed that there was a shoulder on the urea $\delta(\mathrm{NH})$ band at $1545 \mathrm{~cm}^{-1}$ directly proportional to the concentration of spiked albumin and proving the shoulder corresponded to the Amide II of proteins. 
These results demonstrate that ATR FTIR spectra of pre-concentrated extracts can quantify microproteinuria in urine. Nevertheless, two main challenges of this hypothetical method could be anticipated. First, since the dried film contains mainly urea, creatinine and other metabolites, the absorbance of the 1545 $\mathrm{cm}^{-1}$ shoulder is related to the relative concentration of proteins to the other components instead of having a linear relationship with the real concentration in the original urine sample. Secondly, the protein signals are obscured by the urea bands, and it is difficult to extract information about the nature of the protein causing the microporteinuria. Hence, a washing procedure was introduced. When $450 \mu \mathrm{L}$ of ultrapure water was added to the pre-concentrated extract, the metabolites and proteins were diluted 22 times. After ultracentrifugation, the proteins were again pre-concentrated but the non-proteic molecules remained diluted. Thus, the resulting extract contained 22 times more proteins and 22 times less non-proteic interferences (e.g. urea, creatinine and sulphates). If this process is repeated 3 times, the molecules, which are not retained in the filter, are diluted more than 10000 times (see Scheme S1). Figure 1a shows the resulting spectra that display typical features of isolated proteins. Amide A $\left(3303 \mathrm{~cm}^{-1}\right)$, I $\left(1640 \mathrm{~cm}^{-1}\right)$, II $\left(1542 \mathrm{~cm}^{-1}\right)$ and III $\left(1388 \mathrm{~cm}^{-1}\right)$ bands dominate the spectra, indicating proteins of the healthy volunteers were being detected. The intense band in the alcohol region (1200-100 $\left.\mathrm{cm}^{-1}\right)$ is not a typical band from proteins and may be caused by the presence of glycerol remnants or glycoproteins, e.g. transferrin.

\section{LOD, LOQ and reproducibility studies on artificial urine}

The fact that it is possible to measure a clear spectrum of isolated proteins in urine from a healthy volunteer led to the development of a method for detecting and quantifying microporteinuria. From the outset, it was necessary to determine a reproducible relationship (ideally linear) between the protein concentration and the protein bands from the dried film spectra. Given all the urine samples form volunteers contained proteins, it was difficult to establish proper blanks to calculate analytical figures of merit such as the LOD. Thus, artificial urine spiked with albumin was used. Figure 2 shows the spectra at artificial urine spiked with different levels of albumin within the microproteinuria range. It can be clearly seen that the amide bands increase as the concentration of protein increases. We also studied the effect of the ATR correction on the calibration, but the corrected spectra (Figure S1) provided similar results. The integration of the amide bands (1700-1500 $\mathrm{cm}^{-1}$, using baselines fitted on the $1503-1474 \mathrm{~cm}^{-1}$ and $1750-1720 \mathrm{~cm}^{-1}$ regions) were used for 
computing a univariate calibration (see Figure $2 b$ ). The integrated area was found to be linear with the spiked concentration in the microalbuminuria range of concentrations $\left(\mathrm{R}^{2}=0.97\right)$. The LOD, calculated as the concentration corresponding to three times the standard deviation of the blanks was found to be 6.7 p.p.m. (LOQ $22 \mathrm{ppm}$ ). The blanks used were pre-concentrated extracts of artificial urine without spiking, and a visual inspection of the spectra revealed contaminations of glycerol and other components (See Figure S2). Although the intensities are negligible compared with microporteinuria concentration levels, the presence of these bands increased the standard deviation of the blank signals and raised the LOD. For the control blanks, distilled water was dried onto an ATR crystal and the LOD was calculated to be $1 \mathrm{ppm}$. Hence, glycerol contamination is affecting the sensitivity of the method and there is still room for improvement for achieving lower detection limits. Nevertheless, the calculated LOQ is below the lower critical value for microproteinuria diagnosis (20 ppm). Regarding reproducibility, Relative Standard Deviation (RSD\%) values of 8.5, 8.1, 6.9, 6.2 and 14\%, were found for the 20,50,100, 200 and $200 \mathrm{ppm}$ solutions, respectively. These values refer to three replicates of each measurement and on three calibrations obtained on different days $(n=9)$.
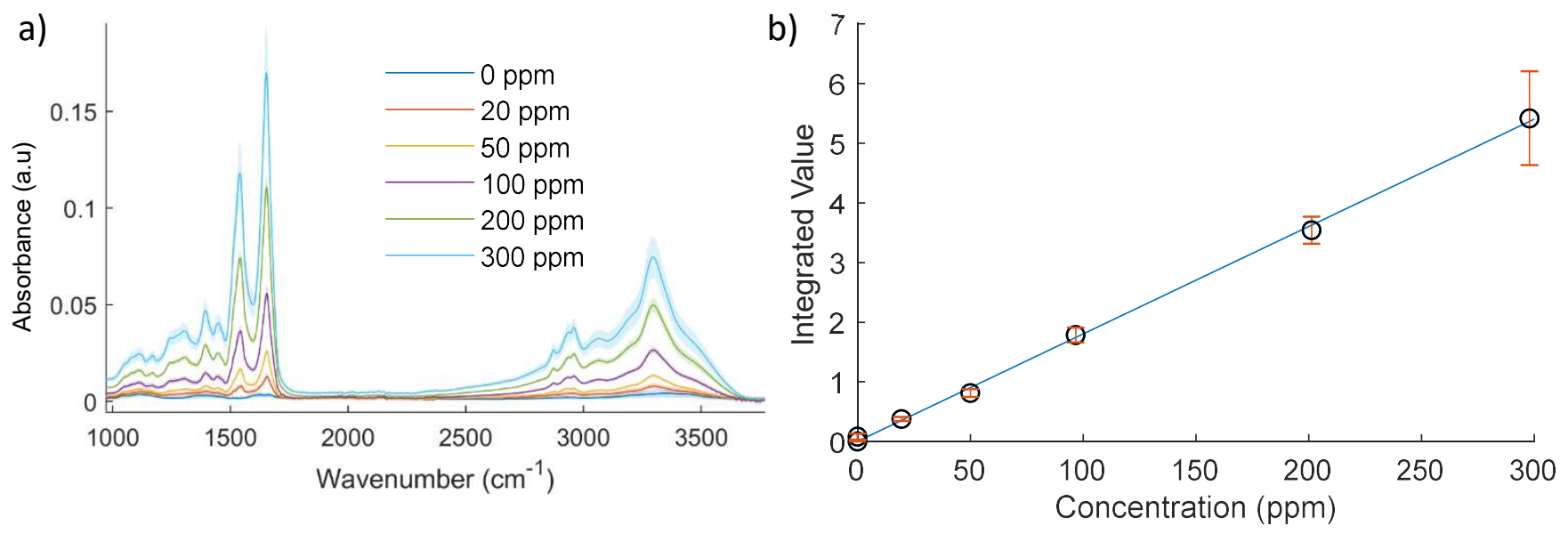

Figure 2. (a) Average spectra of proteic extracts from artificial urine spiked with different amounts of albumin within the microalbuminuria range. (b) Calibration obtained using the integrated area under the Amide I and II bands. Error bars indicates confidence intervals for a significance level of $0.05(n=9)$. 
The linearity of the absorbance with the concentration found in the previous section raised a question about the morphology of the dried films. According to the Beer-Lambert Law, absorbance of an analyte is $\left(\right.$ approximately ${ }^{28}$ ) directly proportional with the concentration of the specific compound and the pathlength. The spectra in Figure 2 indicate that dried films are almost entirely composed of proteins, so the concentration of proteins can be considered as constant. This is a very different scenario from previous work on dried films measured in transmission, where the concentration of dried films is variable and internal standards such as KSCN are used to compensate for changes in the pathlength ${ }^{29,30}$. In our study, the linearity of the signal with the concentration of the solution observed in Figure 2 can only be caused by changes in the pathlength. This can be explained if we consider that, as long as the volume deposited is constant, the amount of proteins deposited on the ATR crystal is proportional to the concentration of proteins in solution. Considering a $0.8 \mu \mathrm{L}$ deposit of protein, concentrations of 20, 50, 100, 200 and $300 \mathrm{ppm}\left(\mathrm{mg} \mathrm{L}^{-1}\right)$ will lead to depositions of 16, 40, 80,160 and $240 \mathrm{pg}$. The method can only be linear if this increase of mass involves a proportional change in the pathlength, i.e. the dried film occupies more of the active volume of the sensor. Considering the complex behaviour of the evanescent wave in an ATR crystal, the linearity will depend on the morphology and reproducibility of the dried film created. As this is of critical concern for the proposed method, we performed an investigation into dried films using imaging techniques.

Figures $3 \mathrm{a}$ and $3 \mathrm{~b}$ show spectra of urine from a healthy volunteer spiked with 50, 150, 300, 500, 600, 800, $1000 \mathrm{ppm}$ of albumin. The integrated area of the amide I and II bands increased linearly in the lower and medium concentration range. Above $600 \mathrm{ppm}$ the sensitivity starts to decrease indicating saturation. Dry films from the calibration solutions were studied using a visible microscope. Figure $3 \mathrm{c}$ shows the visible images of the dry films obtained from drying the extracts of the spiked urine on an ATR diamond crystal. It can be seen that the dried film has a ring shape, and the metallic boundaries fixing the ATR crystal confined the droplet on the crystal, ensuring that the evanescent beam interrogated the whole of the deposited mass. The ring is caused by the so-called coffee ring effect, which has been previously described ${ }^{31}$. This is depicted in the Figure 3d, which shows a graphical representation of the drop on the dried surface of the ATR crystal. Figure 3c indicates that in the range between 50 and $500 \mathrm{ppm}$, as the spiked concentration of albumin is increased, the amount of proteins observable on the centre of the ring increased. After $500 \mathrm{ppm}$, the centre of the ring is completely covered by proteins. The fact that this concentration point roughly corresponds to the saturation 
of the signal (Figure 3a) suggests that the absorbance increases on increasing the amount of proteins in the centre of the ring, and it saturates once all the ring is covered.

Dry films reported in previous serum studies from Hughes et $\mathrm{al}^{32,33}$ showed a different shape, containing much more proportion of the absorbance in the middle of the ring. For example, the deposition of $1 \mu \mathrm{L}$ of the 10-100 KDa fraction of serum resulted in a homogenous thick film being formed, as opposed to a ring. The singular shape of the urine dried films appears to be the result of the low concentration of proteins in the preconcentrated urine extracts (600-6000 ppm) compared with the high concentration of proteins in serum (6$8 \%)$.
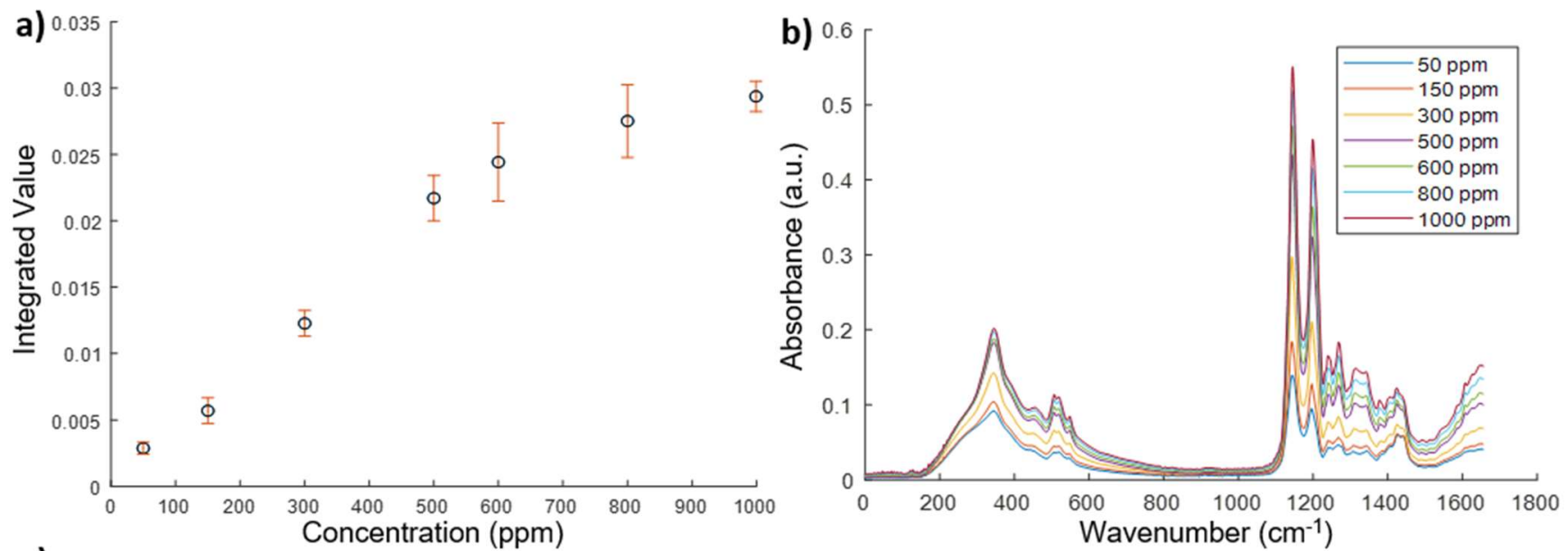

c)

50 ppm

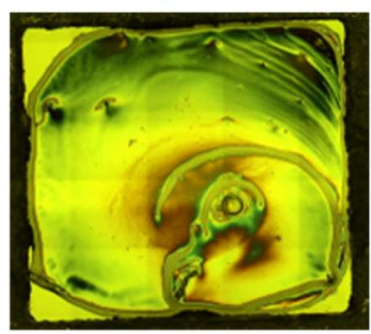

300 ppm

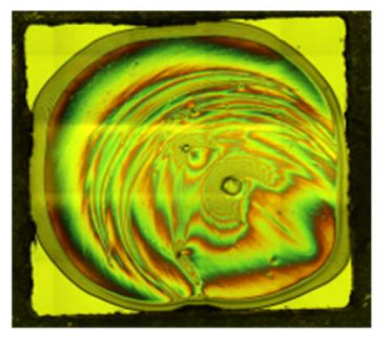

800 ppm
150 ppm

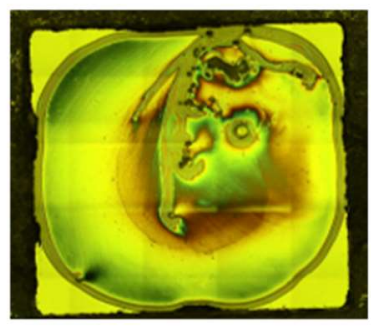

500 ppm

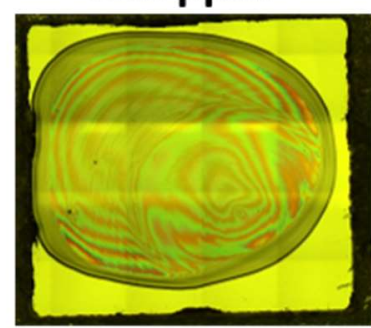

d)
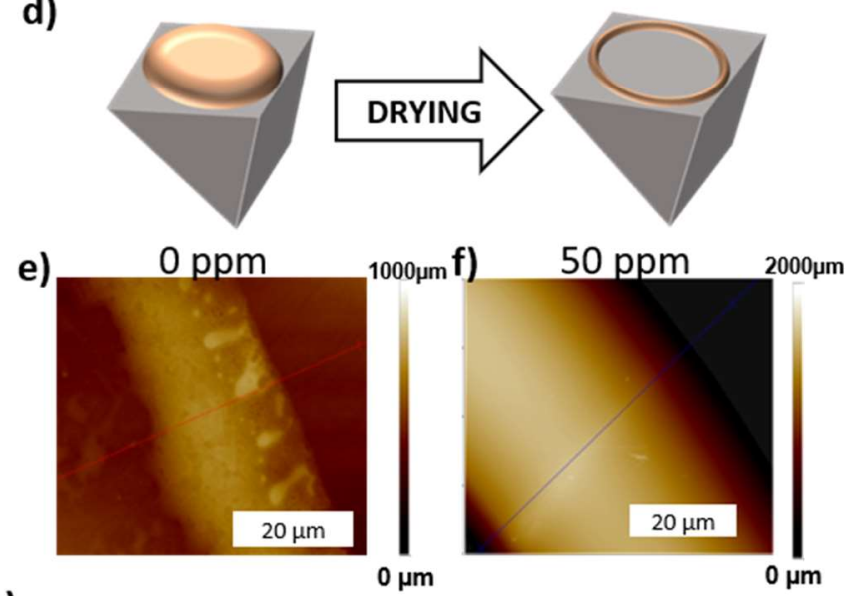

g)

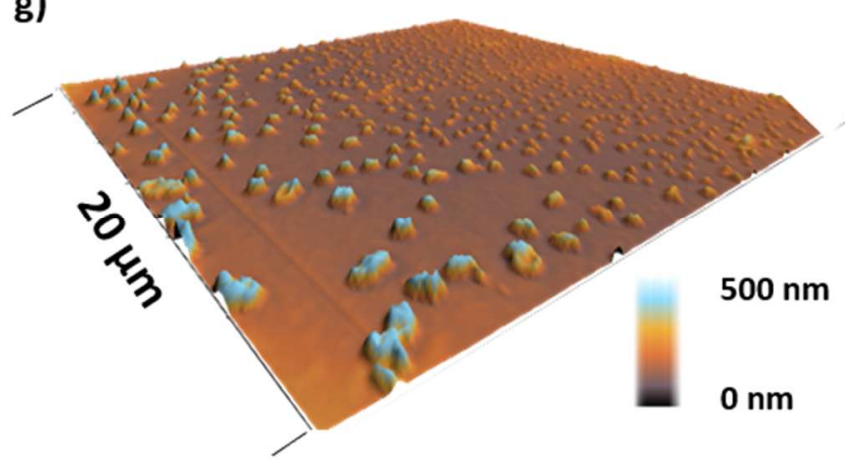


Figure 3. Study of dried films. (a) Calibration curve of urine spiked with albumin (Amide I and II bands). Error bars represent the confidence interval for a significance level of 0.05. (b) Average spectra of the 4 replicate spectra of dried films measured by ATR-FTIR spectroscopy. (c) Visible images of the dry films on an ATR crystal obtained from the extracts of urine spiked at different concentrations (d) Diagram showing the drying process (e) and (f) AFM topographical images of the rings formed by extracts of artificial urine spiked with 0 and 50 ppm, respectively. Profiles are available in Figure S3. (g) AFM image of the middle of the ring of the dried film created after dried in a 1000 ppm albumin solution.

AFM was used to study the thickness of the ring. As it was impossible to place the plate of the ATR under the AFM stage, this study was performed on $\mathrm{CaF}_{2}$ substrate. The penetration depth of the evanescent field through a diamond ATR crystal at 45 degrees and a refractive index of 1.5 for the dry film, ranges from 0.5 to 5 microns in the $4000-400 \mathrm{~cm}^{-1}$ range. In the amide I and II region $\left(1800-1400 \mathrm{~cm}^{-1}\right)$, where the calibrations were performed between 1-1.3 $\mu \mathrm{m}$ (Calculated using equation available on Figure S1). For a blank of artificial urine, the thickness of the dried film was already above $1 \mu \mathrm{m}$ (See figure $3 \mathrm{~d}$ ). For low concentrations such as $50 \mathrm{ppm}$, the thickness was above $2 \mu \mathrm{m}$ (Figure 3e) and rings from dried films of 100 and $150 \mathrm{ppm}$ also had a thickness above this $2 \mu \mathrm{m}$. AFM profiles are available in Figure S3. Thus the contribution of the height to the spectra should be the same for all concentrations above $50 \mathrm{ppm}$ and thus, the linearity cannot be given by the ring thickness. AFM images from the central region of the dried films corresponding to low concentrated solutions revealed that there are small depositions of proteins with a thickness below the penetration depth of the ATR (See Figure 3g). This is consistent with the AFM images for 50-150 ppm in Figure 3a. providing evidence that the amount and distribution of these small depositions could explain the linearity. It should be noted that this $\mathrm{AFM}$ analysis was performed on $\mathrm{a} \mathrm{CaF}_{2}$ substrate, and the drying of the extracts can present differences to the dry rings obtained on diamond.

In summary, the data suggested that the linearity between the absorbance and the protein concentration observed empirically could be caused by an increase in the surface area of the ATR crystal covered by the protein deposit. We conclude that further studies are needed to understand more about the morphology of the 
dried film, which will help to create optimised films, which homogeneously distribute the proteins on the ATR crystal, increasing sensitivity and selectivity.

\section{Spiking of human urine samples}

Artificial urine does not contain all of the components and cell types found in human urine and hence the method was further evaluated using urine samples from 6 healthy volunteers spiked with albumin, haemoglobin and immunoglobulin, at microproteinuria levels. Values of the integrated areas of the amide I and II bands plotted against the spiked concentration are shown in Figure 4. It can be seen that the absorbance was linear with the spiked concentration in the microproteinuria range. The spectral bands from proteins were detected in all urine from the healthy volunteers, indicating that the method had enough sensitivity to detect proteins in urine from healthy individuals. A simultaneous calibration model using artificial urine was created in parallel for each calibration, which was used to calculate the protein concentration in each solution. For evaluating the accuracy of the method, the estimated spiked concentrations were calculated by computing the concentration of the solution and then subtracting the estimated concentration of the corresponding un-spiked urine. A comparison of the estimated values and the spiked values (See Figure S4) was used to compute the RMSEP, which was found to be $23 \mathrm{ppm}$ 

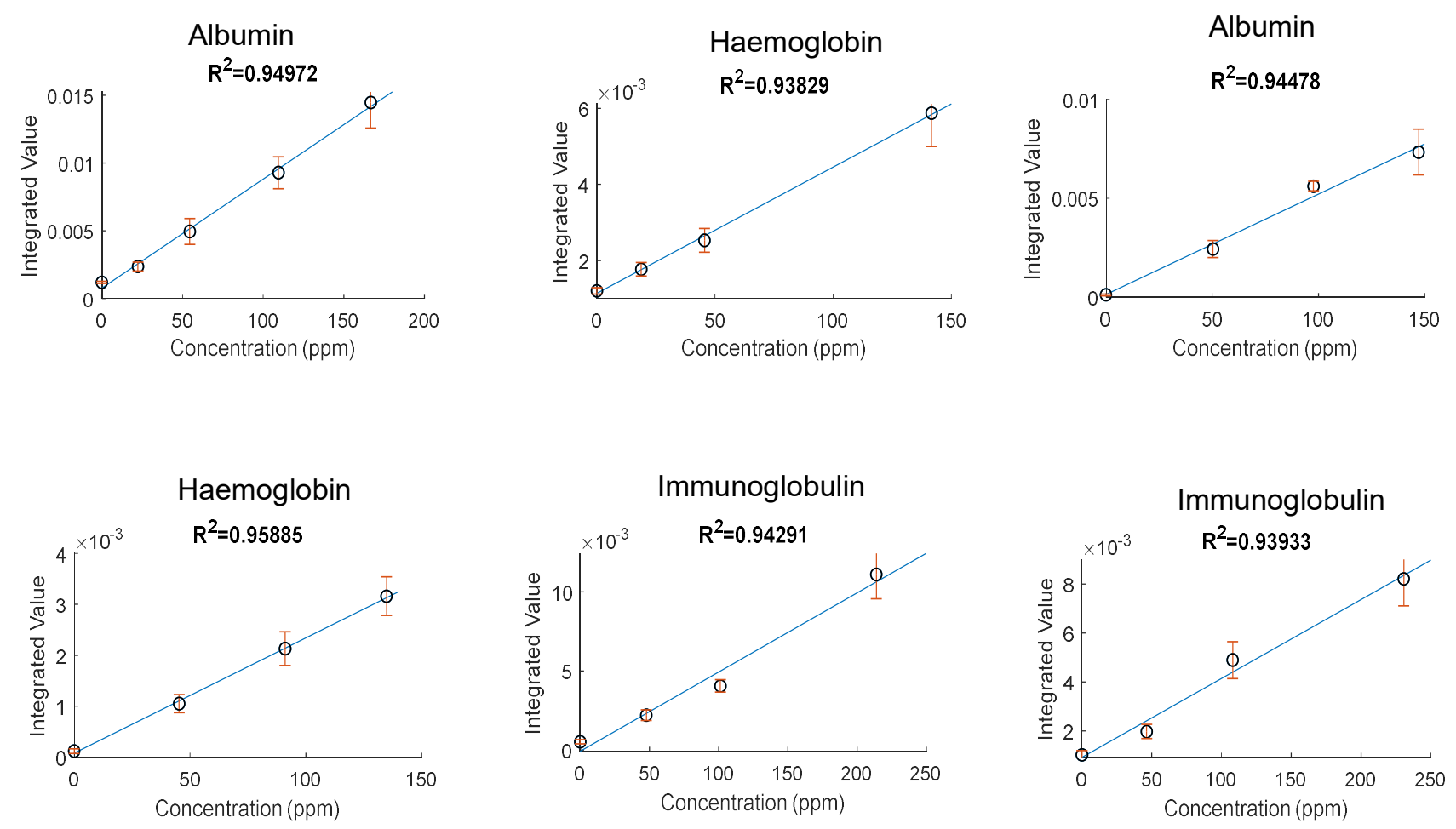

Figure 4. Calibration curves for the 6 different urine samples spiked with albumin, haemoglobin or immunoglobulin at different concentrations. The integrated value refers to the area under the Amide I and II bands.

Identification of the proteins causing the microproteinuria

Each protein shows different spectral patterns caused by differences in both the protein secondary structure and the amino acid composition. ${ }^{34,35}$ These specific spectral patterns can be exploited by multivariate analysis to develop classification models, which can distinguish the protein causing the microproteinuria. In a simple scenario where microproteinuria is caused by a single protein (i.e. the other proteins remain below the normal cut-off), multivariate analysis could use the specific spectral features of proteins to detect the protein causing the microproteinuria. The dataset from the previous section from healthy urine spiked with different amounts of hemoglobin, albumin and immunoglobulin was used to identify the specific protein spiked into the urine. To correct for confounding effects such as the concentration, spectra were normalized using SNV. The PCA scores plot (Figure 5a) showed that the spectra were clustered according to the specific protein contained in the sample. 
A more complex problem is the detection of different proteins when they are above the microproteinuria level. The extracted spectra will be a combination of contributions from the different major proteins, and multivariate analysis needs to decompose the spectra among their different constituents. To test this scenario, a co-spiking experiment was carried out combining spiking 18 aliquots from a volunteer urine sample with 0 , 50,150 and $300 \mathrm{ppm}$ of albumin and immunoglobulin and 0 and $50 \mathrm{ppm}$ of haemoglobin (See Table S1). The haemoglobin was kept to lower levels to investigate minor contributors to the spectra.

MCR-ALS analysis was applied to this dataset using non negativity constraints in both, concentrations and spectra. Singular Value Decomposition (SVD) indicated 4 components. Figure 5a compares the pure spectra of the first three components extracted by MCR-ALS while the fourth component, shown in Figure S5, represented non proteic urine components) with standard spectra of the proteins assigned. It can be seen that spectral profiles are similar to the standard spectra, with correlation coefficient values of $0.9,0.97$ and 0.97 for immunoglobulin, hemoglobin and albumin, respectively. Concentration profiles (See Figure S6) indicated a clear correlation between the concentration spiked of albumin and immunoglobulin and the relative concentration extracted by the MCR-ALS. Nevertheless, for hemoglobin the method was not able to discriminate between the 0 and $50 \mathrm{ppm}$, indicating that the multi-quantification of several proteins can be problematic at low concentration levels. A deeper study of the multicomponent prediction of proteins is out of the scope of this study but future studies on human urine will improve the robustness of the modelling. 

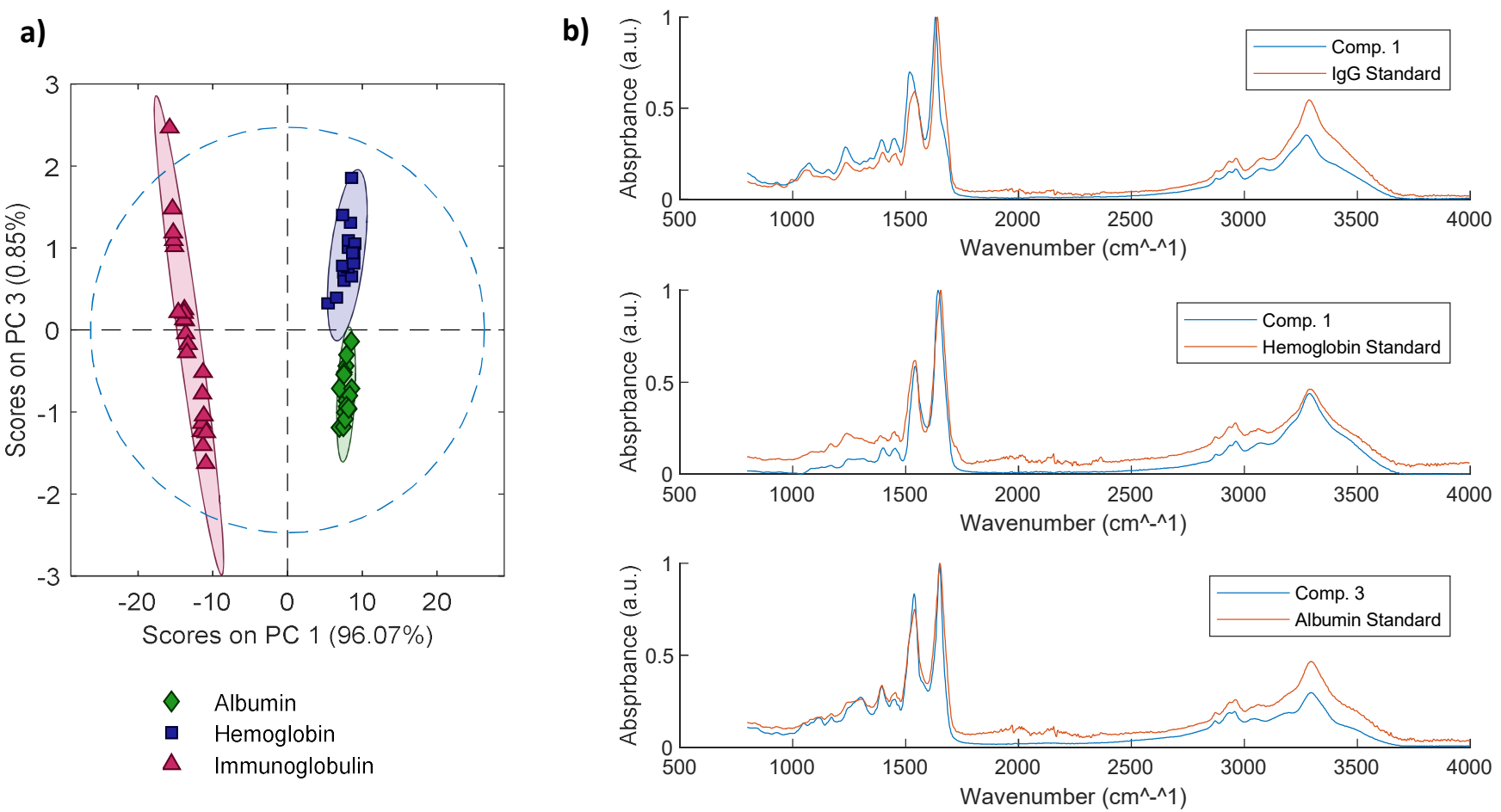

Figure 5. (a) Scores plot showing the clustering of spectra according to the type of protein that was spiked into the urine. Each point represents the spectrum of a urine sample spiked with albumin, haemoglobin or immunoglobulin in the microproteinuria range. (b) Standard spectra of proteins and pure spectra extracted using $M C R-A L S$

\section{Conclusions}

The results show that the combination of ultrafiltration and ATR-FTIR spectroscopy of dried films provides a method for the quantification of proteins in the microproteinuria range. The pre-concentration and washing steps isolate the proteins from other major components in urine. This enables the deposition of highly pure proteins onto the ATR crystal. These proteins can be quantified because the absorbance of the proteins is linear with the concentration of proteins in the solution. Images indicated that the dried films form a ring instead of a thin homogeneous film. The linearity of Amide I and II band absorbance with protein 
concentration in the deposited solution is caused by the amount of mass deposited on the crystal being directly proportional to the concentration of proteins in the original solution. In addition, the differences in the ATRFTIR spectra of each protein provided information on the type of protein spiked into the urine. The identification of a single protein causing microalbuminuria was straight forward, but the multicomponent detection and quantification of several proteins was challenging for low levels of microalbuminuria.

Nevertheless, there are more challenges to be solved before the translation of the methodology to the clinic. The sample preparation step can be simplified by reducing the number of washing steps, which can be achieved using ultracentrifugation devices with a larger volume. The reproducibility of the deposition can be also improved by using an automated micro-dispenser. Although, the aim of this paper is to present and discuss the analytical capabilities of the method, the next step is to evaluate it under clinical conditions, and we are currently proceeding with a clinical study using urine from diabetic patients. The proposed method shows vast potential in the diagnosis and prognosis of several illnesses, which affect the protein composition of urine, and may provide a versatile tool for the quantification and identification of microporteinuria in the future.

\section{Acknowledgments}

This work was funded by an Australian Research Council Discovery Project DP 3921629 and Australian Research Council Future Fellowship grant FT 120100926. DPG acknowledges support from the European Research Council MSCA grant (Spectro-metrics, 020-MSCA-IF-2017 Project ID:796287)

\section{Supporting Information}

Additional information about the composition of Urine, ATR correction, blanks, dry film topography, cospiking experiment and the Multivariate models performed on the study (PDF).

\section{References}


(1) Simerville, J. A.; Maxted, W. C.; Pahira, J. J. Urinalysis: A Comprehensive Review. Am. Fam. Physician 2005, 71 (6), 1153-1162.

(2) Koroshi, A. Microalbuminuria, Is It so Important? Hippokratia 2007, 11 (3), 105-107.

(3) Jørgensen, L.; Heuch, I.; Jenssen, T.; Jacobsen, B. K. Association of Albuminuria and Cancer Incidence. J. Am. Soc. Nephrol. JASN 2008, 19 (5), 992-998. https://doi.org/10.1681/ASN.2007060712.

(4) Diercks, G. F.; Van, A. B.; Hillege, J. L.; De, P. J.; Rouleau, J. L.; Van, W. G. The Importance of Microalbuminuria as a Cardiovascular Risk Indicator: A Review. Can. J. Cardiol. 2002, 18 (5), 525-535.

(5) Pontremoli, R. Patient with Hypertension and Microalbuminuria. In Hypertension and Renal Organ Damage; Pontremoli, R., Ed.; Practical Case Studies in Hypertension Management; Springer International Publishing: Cham, 2018; pp 15-28. https://doi.org/10.1007/978-3-319-56408-1_2.

(6) Gao, Y. Urine Is a Better Biomarker Source Than Blood Especially for Kidney Diseases. In Urine Proteomics in Kidney Disease Biomarker Discovery; Gao, Y., Ed.; Springer Netherlands: Dordrecht, 2015; Vol. 845, pp 3-12. https://doi.org/10.1007/978-94-017-9523-4_1.

(7) D'Costa, J. J.; Goldsmith, J. C.; Wilson, J. S.; Bryan, R. T.; Ward, D. G. A Systematic Review of the Diagnostic and Prognostic Value of Urinary Protein Biomarkers in Urothelial Bladder Cancer. Bladder Cancer 2016, 2 (3), 301317. https://doi.org/10.3233/BLC-160054.

(8) Thomas, S.; Hao, L.; Ricke, W. A.; Li, L. Biomarker Discovery in Mass Spectrometry-Based Urinary Proteomics. PROTEOMICS - Clin. Appl. 2016, 10 (4), 358-370. https://doi.org/10.1002/prca.201500102.

(9) Berry, J. Microalbuminuria Testing in Diabetes: Is a Dipstick as Effective as Laboratory Tests? Br. J. Community Nurs. 2003, 8 (6), 267-273. https://doi.org/10.12968/bjcn.2003.8.6.11548.

(10) Perez-Guaita, D.; Garrigues, S.; de la, M.; Guardia. Infrared-Based Quantification of Clinical Parameters. TrAC Trends Anal. Chem. 2014, 62, 93-105. https://doi.org/10.1016/j.trac.2014.06.012.

(11) Perez-Guaita, D.; Marzec, K. M.; Hudson, A.; Evans, C.; Chernenko, T.; Matthäus, C.; Miljkovic, M.; Diem, M.; Heraud, P.; Richards, J. S.; Andrew, D.; Anderson, D.A.; Doerig, C.; Garcia-Bustos, J.; McNaughton, D.; Wood, B.R.; Parasites under the Spotlight: Applications of Vibrational Spectroscopy to Malaria Research. Chem. Rev. 2018, 118 (11), 5330-5358. https://doi.org/10.1021/acs.chemrev.7b00661.

(12) Spalding, K.; Board, R.; Dawson, T.; Jenkinson, M. D.; Baker, M. J. A Review of Novel Analytical Diagnostics for Liquid Biopsies: Spectroscopic and Spectrometric Serum Profiling of Primary and Secondary Brain Tumors. Brain Behav. 2016, 6 (9), e00502. https://doi.org/10.1002/brb3.502.

(13) Goodacre, R.; Sergo, V.; Barr, H.; Sammon, C.; Schultz, Z. D.; Baker, M. J.; Graham, D.; Marques, M. P.; SuléSuso, J.; Livermore, J.; Faulds, K.; Sinjab, F.;Campbell, J.; Dluhy, R.; Gardner, P.; Phillips, C.; Diem, M.; Wood, B.; Apolonosky, A.; Karzarian, S.; Fullwood, L.; Gough, K.; Petrich, W.; Lloyd, G.; Ibrahim, O.; Cinque, G.; Sockalingum, G.; Stone, N.; Kendall, C.; McAughtrie, S.; Perez-Guaita, D.; Clark, L.; Gerwert, K.; Bonifacio, A.; Lasch, P.; Bhargava R.; Lepert, G; Mader, K.; Clinical Spectroscopy: General Discussion. Faraday Discuss. 2016, 187, 429-460. https://doi.org/10.1039/C6FD90013E.

(14) Heise, H. M.; Voigt, G.; Lampen, P.; Küpper, L.; Rudloff, S.; Werner, G. Multivariate Calibration for the Determination of Analytes in Urine Using Mid-Infrared Attenuated Total Reflection Spectroscopy. Appl. Spectrosc. 2001, 55 (4), 434-443.

(15) Hosafci, G.; Klein, O.; Oremek, G.; Mantele, W. Clinical Chemistry without Reagents? An Infrared Spectroscopic Technique for Determination of Clinically Relevant Constituents of Body Fluids. Anal. Bioanal. Chem. 2007, 387 (5), 1815-1822.

(16) Oliver, K. V.; Vilasi, A.; Maréchal, A.; Moochhala, S. H.; Unwin, R. J.; Rich, P. R. Infrared Vibrational Spectroscopy: A Rapid and Novel Diagnostic and Monitoring Tool for Cystinuria. Sci. Rep. 2016, 6, 34737. https://doi.org/10.1038/srep34737.

(17) Shaw, R. A.; Low-Ying, S.; Leroux, M.; Mantsch, H. H. Toward Reagent-Free Clinical Analysis: Quantitation of Urine Urea, Creatinine, and Total Protein from the Mid-Infrared Spectra of Dried Urine Films. Clin. Chem. 2000, 46 (9), 1493-1495.

(18) Perez-Guaita, D.; Ventura-Gayete, J.; Pérez-Rambla, C.; Sancho-Andreu, M.; Garrigues, S.; de la Guardia, M. Evaluation of Infrared Spectroscopy as a Screening Tool for Serum Analysis. Impact of the Nature of Samples Included in the Calibration Set. Microchem. J. 2013, 106 (0), 202-211.

https://doi.org/10.1016/j.microc.2012.06.016.

(19) Rohleder, D.; Kocherscheidt, G.; Gerber, K.; Kiefer, W.; Kohler, W.; Mocks, J.; Petrich, W. Comparison of MidInfrared and Raman Spectroscopy in the Quantitative Analysis of Serum. J. Biomed. Opt. 2005, 10 (3), 031108.

(20) Khaskheli, A. R.; Sirajuddin; Sherazi, S. T. H.; Mahesar, S. A.; Kandhro, A. A.; Kalwar, N. H.; Mallah, M. A. Estimation of Ibuprofen in Urine and Tablet Formulations by Transmission Fourier Transform Infrared Spectroscopy by Partial Least Square. Spectrochim. Acta Part-Mol. Biomol. Spectrosc. 2013, 102, 403-407. https://doi.org/10.1016/j.saa.2012.10.021. 
(21) Pérez-Guaita, D.; Sánchez-Illana, Á.; Garrigues, S.; de la Guardia, M. Determination of Lidocaine in Urine at Low Ppm Levels Using Dispersive Microextraction and Attenuated Total Reflectance-Fourier Transform Infrared Measurements of Dry Films. Microchem. J. 2015, 121, 178-183.

(22) Bonnier, F.; Petitjean, F.; Baker, M. J.; Byrne, H. J. Improved Protocols for Vibrational Spectroscopic Analysis of Body Fluids. J. Biophotonics 2014, 7 (3-4), 167-179. https://doi.org/10.1002/jbio.201300130.

(23) Bonnier, F.; Blasco, H.; Wasselet, C.; Brachet, G.; Respaud, R.; Carvalho, L. F. C. S.; Bertrand, D.; Baker, M. J.; Byrne, H. J.; Chourpa, I. Ultra-Filtration of Human Serum for Improved Quantitative Analysis of Low Molecular Weight Biomarkers Using ATR-IR Spectroscopy. The Analyst 2017, 142 (8), 1285-1298. https://doi.org/10.1039/C6AN01888B.

(24) Elsohaby, I.; McClure, J. T.; Riley, C. B.; Bryanton, J.; Bigsby, K.; Shaw, R. A. Centrifugal Ultrafiltration of Human Serum for Improving Immunoglobulin A Quantification Using Attenuated Total Reflectance Infrared Spectroscopy. J. Pharm. Biomed. Anal. 2018, 150, 413-419. https://doi.org/10.1016/j.jpba.2017.12.031.

(25) Worramongkona, P.; Seeda, K.; Phansomboon, P.; Ratnarathorn, N.; Chailapakul, O.; Dungchai, W. A Simple Paper-Based Colorimetric Device for Rapid and Sensitive Urinary Oxalate Determinations. Anal. Sci. Int. J. Jpn. Soc. Anal. Chem. 2018, 34 (1), 103-108. https://doi.org/10.2116/analsci.34.103.

(26) Jaumot, J.; Gargallo, R.; de Juan, A.; Tauler, R. A Graphical User-Friendly Interface for MCR-ALS: A New Tool for Multivariate Curve Resolution in MATLAB. Chemom. Intell. Lab. Syst. 2005, 76 (1), 101-110. https://doi.org/10.1016/j.chemolab.2004.12.007.

(27) Oliver, K. V.; Maréchal, A.; Rich, P. R. Effects of the Hydration State on the Mid-Infrared Spectra of Urea and Creatinine in Relation to Urine Analyses. Appl. Spectrosc. 2016, 70 (6), 983-994. https://doi.org/10.1177/0003702816641263.

(28) Mayerhöfer, T. G.; Popp, J. Beer's Law - Why Absorbance Depends (Almost) Linearly on Concentration. ChemPhysChem 2019, 20 (4), 511-515. https://doi.org/10.1002/cphc.201801073.

(29) Zhou, Y.-P.; Xu, L.; Tang, L.-J.; Jiang, J.-H.; Shen, G.-L.; Yu, R.-Q.; Ozaki, Y. Determination of Glucose in Plasma by Dry Film-Based Fourier Transformed-Infrared Spectroscopy Coupled with Boosting Support Vector Regression. Anal. Sci. Int. J. Jpn. Soc. Anal. Chem. 2007, 23 (7), 793-798.

(30) Shaw, R. A.; Kotowich, S.; Leroux, M.; Mantsch, H. H. Multianalyte Serum Analysis Using Mid-Infrared Spectroscopy. Ann. Clin. Biochem. 1998, 35, 624-632.

(31) Deegan, R. D.; Bakajin, O.; Dupont, T. F.; Huber, G.; Nagel, S. R.; Witten, T. A. Capillary Flow as the Cause of Ring Stains from Dried Liquid Drops. Nature 1997, 389 (6653), 827-829. https://doi.org/10.1038/39827.

(32) Hughes, C.; Brown, M.; Clemens, G.; Henderson, A.; Monjardez, G.; Clarke, N. W.; Gardner, P. Assessing the Challenges of Fourier Transform Infrared Spectroscopic Analysis of Blood Serum: Assessing the Challenges of FTIR Spectroscopic Analysis of Blood Serum. J. Biophotonics 2014, 7 (3-4), 180-188. https://doi.org/10.1002/jbio.201300167.

(33) Hughes, C.; Clemens, G.; Bird, B.; Dawson, T.; Ashton, K. M.; Jenkinson, M. D.; Brodbelt, A.; Weida, M.; Fotheringham, E.; Barre, M.; Rowlette, J.; Baker, M.J.; Introducing Discrete Frequency Infrared Technology for High-Throughput Biofluid Screening. Sci. Rep. 2016, 6 (1). https://doi.org/10.1038/srep20173.

(34) Perez-Guaita, D.; Ventura-Gayete, J.; Pérez-Rambla, C.; Sancho-Andreu, M.; Garrigues, S.; de la Guardia, M. Protein Determination in Serum and Whole Blood by Attenuated Total Reflectance Infrared Spectroscopy. Anal. Bioanal. Chem. 2012, 404 (3), 649-656. https://doi.org/10.1007/s00216-012-6030-7.

(35) Barth, A. Infrared Spectroscopy of Proteins. Biochim. Biophys. Acta-Bioenerg. 2007, 1767 (9), 1073-1101. 